\title{
Sistematización de una estrategia metodológica: intervención físico - psico-afectivo en el adulto mayor desarrollada por los estudiantes de enfermería, cohorte 2018 - 2020.
}

\section{Información de artículo:}

Recibido: 30/09/2021

Aprobado: 15/12/2021

Palabras claves:

Estrategia, Extentension, Metodología, intervención, salud, vida

\section{Keywords:}

Strategy, Extentension, Methodology, intervention, health, life

\section{Resumen}

El presente artículo de investigación tiene como propósito la sistematización de una estrategia metodológica de aprendizaje basado en problemas y materializada por los estudiantes de la carrera de Enfermaría. La estrategia fue diseñada en un Intervención Físico - Psico-afectivo en el adulto mayor desarrollada en tres cohortes según año académico 2018, 2019,2020. El objetivo es fundamental fue promoción de la salud, prevención del daño y mejoramiento de la calidad de vida, de una población vulnerable de sufrir daños en su salud, partiendo de los problemas de la edad se estableció un pan de asistencia para potenciar la movilidad, adiestramiento para mejorar el auto cuido, se involucró al familiar tutor, esta estrategia también contenía acciones estimulación motora, cognitiva y Piscoafectiva, entendiendo como una población que carece en su mayoría de aspectos elementales socio afectivos. En el trayecto de la intervención se manifestaron aspectos interesantes, tales como: fortalecimiento de la acción extensionista de la Universidad, reconocimiento social, desarrollo de capacidades resolutivas y humanísticas por parte de los estudiantes pero también innovación y creatividad de la práctica docente. La información utilizada fue obtenida mediante una base de datos establecida por los estudiantes por cada cohorte la que sirvió como evidencia para demostrar que la estrategia ha sido generadora de impacto social pero también enriquecedor de una actividad extensionista, creativa e innovadora.

\footnotetext{
${ }^{1}$ Máster en Educación e Intervención Social. Profesora de la Universidad Nacional Autónoma de Nicaragua - UNAN-MANAGUA. Email: zenaydao307@gmail.com (D): https://orcid.org/oooo-0002-9582-797X

2 Licenciado en Enfermería con Mención en Materno Infantil. Profesor de la Universidad Nacional Autónoma de Nicaragua - UNAN-MANAGUAEmail: Obandopablog5@gmail.com (D): https://orcid.org/o0oo-0003-0599-4545
} 


\title{
Systematization of a methodological strategy: physical - psychoaffective intervention in the elderly developed by nursing students, cohort 2018 - 2020
}

\begin{abstract}
The purpose of this research article is to systematize a methodological learning strategy based on problems and materialized by the students of the Nursing career. The strategy was designed in a Physical - Psycho-affective Intervention in the elderly developed in three cohorts according to the academic year 2018, 2019, and 2020. The fundamental objective was health promotion, prevention of damage and improvement of the quality of life of a population vulnerable to damage to their health, based on the problems of age, an assistance plan was established to enhance mobility, Training to improve self-care, the family tutor was involved, this strategy also contained motor, cognitive and Pisco-affective stimulation actions, understood as a population that lacks mostly socio-affective elementary aspects. In the course of the intervention, interesting aspects were manifested, such as: strengthening of the extension action of the University, social recognition, development of resolutive and humanistic capacities on the part of the students, but also innovation and creativity of the teaching practice. The information used was obtained through a database established by the students for each cohort, which served as evidence to demonstrate that the strategy has generated social impact but also enriching an extension, creative and innovative activity.
\end{abstract}

\section{Introducción}

El proceso de enseñanza aprendizaje está vinculado a múltiples tareas en el cual convergen diferentes actores, el profesorado constituye una entidad facilitadora que garantiza la dirección de este proceso, alumnos y maestros se convierten protagonistas activos, que construyen, conocimiento y desarrollan habilidades. Las instituciones de educación superior están comprometidas con la sociedad, formando personas integrales con capacidad de resolver problemas sentidos por las poblaciones más vulnerables, con capacidades innovadoras para el desarrollo social sostenible.

Además de la formación las universidades están llamadas a interactuar con la población con diferentes actividades que beneficien a la población, la principal tara es la extensión universitaria maestros y alumnos hacen su marcha fuera de las aulas de clases para dar su aporte tangible vinculante con otras entidades que también convergen en la población. Existen diferentes formas de incidir en la población desde el campo de la docencia, aplicar estrategias metodológicas innovadoras con visión de impacto positivo garantiza la confianza y el prestigio de una unidad académica 
(Pérez, 2016). Documentar una estrategia que sido de ayuda social y reconocida por la misma población proporciona la sistematización del trabajo Académico-formativo tanto dentro como fuera de las aulas de clase.

Por esto hemos sistematizado la estrategia de ayuda aplicada al adulto mayor, con el fin de dar continuidad, hacer ajustes pero además emprender en nuevas estrategias que llegue a impactar positivamente tanto en la práctica académica, en la institución de educación superior, con la formación de personas comprometidas y capaces de hacer transformaciones en la sociedad, pero además lograr incidir en una población que demande de ayuda inmediata como fue el caso de nuestra población meta" los adultos mayores".

\section{Fundamentación Teoríca}

\subsection{Estrategia metodológica de intervención}

El aprendizaje se define como "el proceso por el cual las personas adquieren cambios en su comportamiento, mejoran sus actuaciones, reorganizan su pensamiento o descubren nuevas maneras de comportamiento y nuevos conceptos e información". Las estrategias metodológicas son formas de pensamiento que facilitan el aprendizaje. Establecen un ambiente propicio para el aprendizaje, con respeto y aprecio por la diversidad cultural y lingüística. Permiten pasar de la recepción pasiva de la información a la construcción del conocimiento (Ministerio de Educación de Guatemala, 2010).

En la educación tradicional lo más importante ha sido adquirir la mayor cantidad de conocimientos, en donde el alumno se dedica únicamente a acatar las normas y obediencia al docente mediante cátedra autoritaria oral e inflexible. Este método es totalmente expositivo, mecánico y repetitivo (Garrido, 2015), considera que esta metodología es completamente conductista, consiste en proporcionar contenidos, es decir el alumno recibe e interioriza la información a través de estrategias metodológicas tradicionales diseñadas para transmitir conocimientos de forma pasiva, de manera que esto promueve la memorización teórica, no propician la investigación elemental ni científica, no ayudan a la innovación del conocimiento ni la creatividad.

Vásquez (2010) considera que las nuevas propuestas metodológicas apuestan a que el alumno desarrolle destrezas y habilidades de forma más dinámica y abierta, participativa y colaborativa en el aula de clases, con este se pretende educar al alumno de manera integral. Meledro (2011) manifiesta que una estrategia metodología de intervención aporta al desarrollo de las habilidades, destrezas y construcción del conocimiento de manera práctica e interactiva, de igual forma aporta a una causa social.

El concepto de intervención como estrategia metodológica socio comunitaria es significativo porque se ha caracterizado desde diferentes enfoques teóricos con 
experiencias prácticas muy diversas, implica un esfuerzo de reflexión y de síntesis importante para la práctica docente, construcción de los aprendizajes por los estudiantes y el desarrollo social. De esta afirmación se construye el concepto de acción o intervención socio comunitaria como: "aquella que ayuda a crear escenarios, contextos y proyectos favorecedores del desarrollo y fortalecimiento de los individuos, los colectivos, las organizaciones y las instituciones que configuran una comunidad" (Meledro, 2011).

De aquí parte la necesidad de establecer el cambio de paradigmas del proceso de enseñanza aprendizaje, con estrategias que relacionen el conocimiento con la acción de manera que favorezca la construcción de conocimientos dirigidos a resolver problemas sociales, educativos y culturales para desarrollo humano colectivo, pero ademas valorando aquellas que han dado aporte significativo en el desarrollo humano sostenible de la población, requiere por tanto una rigurosa sistematización que evidencie su implicancia.

\subsection{Sistematización de estrategias de aprendizaje}

La sistematización de estrategias de aprendizaje interpreta la relación de la teoría con la práctica, orientan a comprender el objetivo de su aplicabilidad, aprender de ella como un referente metodológico para la transmisión de los aprendizajes, difusión de información y la reflexión crítica; brinda mayor posibilidad de comprender la diversidad cultural de la población (Zavala, 2010). En FAO (2004) se cita a Martinic (1984) quien hacen referencia que la sistematización es un proceso de reflexión que pretende ordenar u organizar lo que ha sido la marcha, los procesos, los resultados de un proyecto, buscando en tal dinámica las dimensiones que pueden explicar el curso que asumió el trabajo realizado.

Este proceso tiene como propósito la participación interdisciplinaria, multidisciplinaria que supone un acercamiento hacia todos los involucrados en la intervención, especialmente hacia los beneficiarios. Brinda, por tanto, la oportunidad de analizar junto a ellos, mediante el testimonio, los cuidados y su interacción con los factores que contribuyeron positiva o negativamente al desarrollo de la intervención. Una Sistematización permite obtener resultados fidedignos documentados de una experiencia metodológica aplicados, en ella se busca el cambio social desde la práctica. Toda sistematización es en esencia un proceso de aprendizaje y construcción de conocimientos (Pérez, 2016). La extensión critica orienta a la práctica comunitaria desde la acción universitaria por su alto compromiso social, de ella se deriva la interacción cultural, la investigación de campo para la solución de problemas más sentidos por la población más vulnerables (Valenzuela-Tovas, 2018). 


\subsection{Sistematización como aporte a la extensión universitaria.}

La importancia de sistematizar una estrategia de intervención es también organizar y documentar sus aportes en el contexto de su aplicación, uno de estos se marca desde la extensión universitaria como función social de la Universidad, permite desarrollar los procesos evolutivos del sistema educativo como un sistema funcional que interviene en los problemas típicos desde la acción formativa (Cedeño \& Machado, 2012).

La Extensión universitaria transmite el conocimiento y promueve el desarrollo cultural en los distintos sectores de la sociedad comprometida con la problemática social, contribuye a demás a la solución de problemas (Di Mari, 2013). Para Menéndez (2017) La extensión universitaria es considerada en el contexto de las universidades nacionales, como una de las funciones esenciales que conjuntamente con la investigación y la docencia constituyen los pilares básicos sobre los que se construye un modelo de universidad democrática y comprometida socialmente, su compromiso es permanente hacia la excelencia, pertinencia y equidad social.

Ante esta premisa se ha logrado la vinculación de docentes, profesionales y alumnos, dejando de lado las divisiones jerárquicas, trabajando en equipo con un mismo objetivo, aprendiendo uno del otro en beneficio las poblaciones más vulnerables, entendiendo cada problema pero además proponiendo soluciones que garanticen el verdadero cambio para el desarrollo humano individual y colectivo. Las universidades se han convertido en el pilar fundamental en la aplicación de estrategias de solución fuera de las aulas de clase a través de un intercambio con el ceno popular, es ahí que se logra una verdadera interacción social, cultural educativa-formativa.

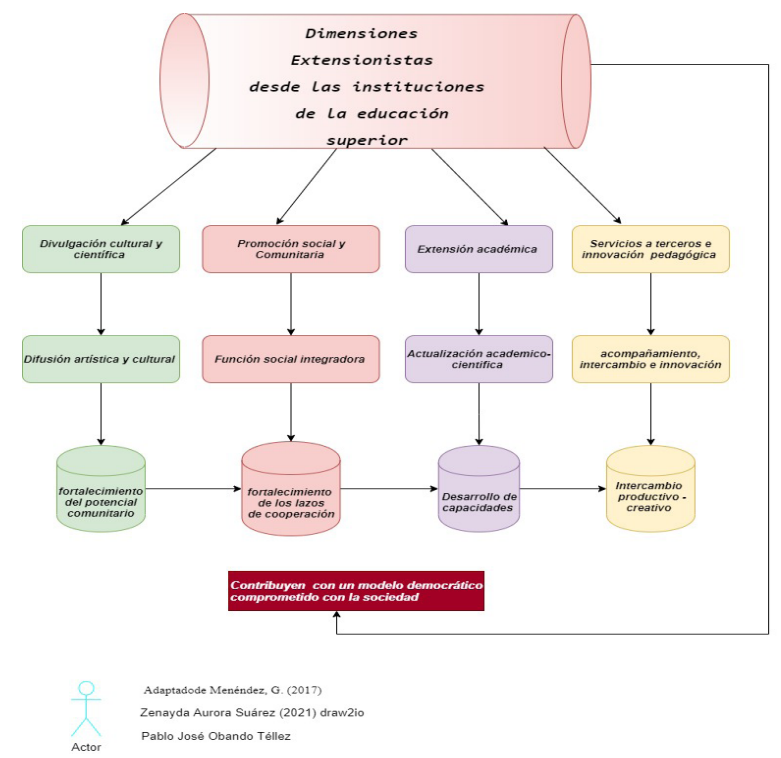

Figura 1: Dimensiones extensionistas desde las instituciones de educación superior 


\section{Propuesta de Intervección}

Objetivo: Sistematizar una estrategia metodológica de Intervención Físico - Psicoafectivo en el adulto mayor desarrollada por los estudiantes de Enfermería.

\begin{tabular}{|c|c|c|c|c|}
\hline Categorías & Descripciones & Sub Categorías & Ítems & Preguntas \\
\hline \multirow[t]{2}{*}{$\begin{array}{l}\text { Práctica acadé- } \\
\text { mica. }\end{array}$} & \multirow{2}{*}{$\begin{array}{l}\text { Es la representación } \\
\text { de las relaciones que } \\
\text { predomina en el acto } \\
\text { de enseñar, es tam- } \\
\text { bién un paradigma } \\
\text { de coexistir con otros } \\
\text { y que sirve para orga- } \\
\text { nizar la búsqueda de } \\
\text { nuevos conocimien- } \\
\text { tos (Pérez, Blanco \& } \\
\text { Gómez, 2017). }\end{array}$} & \multirow[t]{2}{*}{$\begin{array}{l}\text { Docencia } \\
\text { Investigación } \\
\text { Extensión } \\
\text { Oportunidad } \\
\text { Contexto } \\
\text { Propósito }\end{array}$} & \multirow{2}{*}{$\begin{array}{l}\text { Constructo } \\
\text { teórico. }\end{array}$} & $\begin{array}{l}\text { 1. ¿Cuáles son las técnicas metodológicas tradicionales } \\
\text { más usadas en el aula de clases? } \\
\text { 2. ¿Cómo contribuye la extensión universitaria en la gene- } \\
\text { ración de conocimientos del estudiante? } \\
\text { 3. ¿Cómo se vincula la práctica académica con la exten- } \\
\text { sión universitaria? }\end{array}$ \\
\hline & & & & $\begin{array}{l}\text { 4. ¿Cómo vincular un proceso teórico práctico desde la } \\
\text { formación? } \\
\text { 5. ¿Cuál es el espacio idóneo para la ejecución de una } \\
\text { intervención social? } \\
\text { 6. ¿Cuál es el propósito de una intervención social como } \\
\text { una metodología educativa? }\end{array}$ \\
\hline
\end{tabular}

\section{Tabla 2. Sistematización de la estrategia cohorte 2018 - 2020}

\begin{tabular}{|c|c|c|c|c|}
\hline Categorías & Descripciones & Sub Categorías & Ítems & Preguntas \\
\hline $\begin{array}{l}\text { Valoración } \\
\text { Físico - Psico- } \\
\text { afectivo en el } \\
\text { adulto mayor } \\
\text { desarrollada } \\
\text { por los estu- } \\
\text { diantes de } \\
\text { Enfermería }\end{array}$ & $\begin{array}{l}\text { Es un modelo integrador } \\
\text { de ayuda al adulto mayor } \\
\text { que le permite integración } \\
\text { social, reducción de daño, } \\
\text { mantenimiento de la } \\
\text { salud y calidad de vida } \\
\text { (Quintero-Rodríguez, } \\
\text { Victorio-Quintero, \& } \\
\text { Botero-Caicedo, 2015) }\end{array}$ & $\begin{array}{l}\text { Exploración } \\
\text { Selección del } \\
\text { problema }\end{array}$ & $\begin{array}{l}\text { Aprendizaje basado } \\
\text { en problemas. }\end{array}$ & $\begin{array}{l}\text { ¿Cómo selecciono a los protagonistas? } \\
\text { ¿Cuál fue la población asistida? } \\
\text { ¿Cuantos protagonistas fueron asistidos? } \\
\text { ¿Cuál fue el método utilizado en la identificación } \\
\text { de necesidades de ayuda? } \\
\text { ¿Cuáles fueron los principales hallazgos encon- } \\
\text { trados? }\end{array}$ \\
\hline $\begin{array}{l}\text { Evidencia In } \\
\text { situ. }\end{array}$ & $\begin{array}{l}\text { Tiempo real en el que suce- } \\
\text { den las cosas (Indra. S.f.). }\end{array}$ & $\begin{array}{l}\text { Valoración inicial } \\
\text { Intervenciones }\end{array}$ & $\begin{array}{l}\text { 1. Físicos } \\
\text { 2.Cognitivo } \\
\text { 3. Psi-coactivo } \\
\text { 4.Estimulación } \\
\text { motora. } \\
\text { 5.Estimulación } \\
\text { Cognitiva } \\
\text { 6.Acompañamiento } \\
\text { Psico-afectivo }\end{array}$ & $\begin{array}{l}\text { ¿Cómo se manifestaron los problemas físicos en la } \\
\text { salud del adulto mayor? } \\
\text { ¿Cómo se manifestaron los problemas cognitivos } \\
\text { en la salud del adulto mayor? } \\
\text { ¿Cómo se manifestaron los problemas Psico- afec- } \\
\text { tivo en la salud del adulto mayor? } \\
\text { ¿De acuerdo a los hallazgos encontrados cuales } \\
\text { fueron las principales intervenciones de estimula- } \\
\text { ción motoras? } \\
\text { ¿De acuerdo a los hallazgos encontrados cuales } \\
\text { fueron las principales intervenciones de estimula- } \\
\text { ción cognitiva? } \\
\text { ¿De acuerdo a los hallazgos encontrados cuales } \\
\text { fueron las principales intervenciones de acompa- } \\
\text { ñamiento Psico-afectivo? }\end{array}$ \\
\hline
\end{tabular}




\section{Resultados de la Intervección}

\section{Categoría 1. Practica Académica.}

La práctica académica es un espacio de grandes oportunidades, maraca las pautas para incursionar diferentes campos de acción que responden a las necesidades sociales de carácter formativas, investigativas y constructivas en la generación de conocimientos. Tomando como referencia la práctica docente seleccionamos las siguientes categorías: Docencia, Investigación, Extensión, Oportunidad, Contexto y propósito, mismas que le dieron salida a las interrogantes planteadas para la sistematización de la estrategia extensionista ejecutada en 3 momentos eferentes:

1. ¿Cuáles son las técnicas metodológicas tradicionales más usadas en el aula de clases?

Las técnicas metodológicas son expositivas, teórica - conceptual, conferencistas, aprendizaje basado en problemas hipotéticos, memorística y repetitiva.

2. ¿Cómo contribuye la extensión universitaria en la generación de conocimientos del estudiante?

Desde todos los puntos de vista la extensión universitaria contribuye a la generación de Conocimientos y transformación social.

3. ¿Cómo se vincula la práctica académica con la extensión universitaria?

Se vincula directa desde la práctica Profesionalizarte, participación intersectorial, cultural, investigación, acompañamiento técnico.

4. ¿Cómo vincular el proceso teórico práctico con la comunidad?

La investigación, la acción y la participación, vinculan la práctica académica desde las diferentes teorías disciplinares.

5. ¿Cuál es el espacio idóneo para la ejecución de la acción?

Es cualquier contexto en el que intervienen los seres humanos.

6. ¿Cuál es el propósito de una intervención social dentro de una metodología educativa?

Permite la transformación de una situación problema real aun estado de bienestar y confort. 


\section{Categoría 2. Valoración Físico - Psico-afectivo en el adulto mayor}

7. ¿Cómo selecciono a los protagonistas?

Se seleccionó por conveniencia al adulto mayor que tenía algún parentesco con estudiantes de enfermería tercer año que cursaban la asignatura de "Cuidados Geriátricos." En el cual se hizo un paréntesis con el programa de la asignatura que conlleva a la valoración del adulto mayor en asilos de ancianos, entonces fue en este momento que se cambió la estrategia de una técnica de aprendizaje procedimental dirigido al dominio de estructuras teóricas a un proceso de aprendizaje basado en problemas pero participativo- interactivo de acción inmediata.

8. ¿Cuál fue la población asistida?

Se seleccionaron a 15 adultos mayores por cada año $(2018,2019,2020)$

9. ¿Cuantos protagonistas fueron asistidos?

Se logró asistir 45 adultos mayores más 45 tutores, para un total de 90 protagonistas

10. ¿Cuál fue el método utilizado en la identificación del problema?

Para valoración del adulto mayor, se aplicaron diferentes guías ya establecidas y validadas para la atención geriátricas, las que describen a continuación: Guía del examen físico general, valoración de las actividades de las vidas diarias - índice de Kartz para el manejo de la demencia y la independencia, Escala de Lawton Brody para las actividades instrumentales de la vida diaria, Valoración Mini Nutricional Assessment Nestlé, Escala Geriátrica de la depresión.

11.¿Cuáles fueron los principales hallazgos encontrados?

De los 45 protagonistas el 100\% tenían al menos una enfermedad crónica degenerativa (Diabetes, artritis reumatoide, hipertensión arterial, obesidad mórbida). Desde el punto de vista Psico-afectivo el 70\% presentaba sentimiento de soledad, tristeza, incapacidad, un número reducido 30\% presentaba Demencia Senil, Alzheimer, depresión, ansiedad, delirio síndrome confuso y un 100\% las poli farmacias.

\section{Categoría 3. Evidencia In situ.}

12. ¿Cómo se manifestaron los problemas físicos en la salud del adulto mayor? En Cada uno de las cohortes estudiadas la tendencia fue similar, una población afectada por problemas de la edad dentro los más importantes se mencionan los 
siguientes: Deterioro de la movilidad, deformaciones óseas, pérdida del equilibrio, pérdidas de los sentidos sensoriales o disminución de los mismos.

13. ¿Cómo se manifestaron los problemas cognitivos en la salud del adulto mayor?

En su mayoría mostraban deterioro de la memoria retentiva, delirio o síndrome confuso.

14. ¿Cómo se manifestaron los problemas Psico-activo en la salud del adulto mayor?

Un 95\% manifestaba sentimiento de soledad, tristeza, abandono, incapacidad, dependencia progresiva, aunque los tutores mostraban cierto nivel de responsabilidad se percibían desprotegidos en algún momento.

15. ¿De acuerdo a los hallazgos encontrados cuales fueron las principales intervenciones de estimulación motoras?

Desde el punto de vista de la movilidad fueron: Aplicación y enseñanza de algunos ejercicios físicos de las extremidades inferiores y superiores, estimulación de la deambulación y control del reposo.

16. ¿De acuerdo a los hallazgos encontrados cuales fueron las principales intervenciones de estimulación cognitiva?

Aplicación de herramientas para la estimulación del recuerdo (utilización de álbum de fotografía familiar, repertorios musicales de su época, eventos especiales como bodas, bautismos, también utilización de rompecabezas simples).

17. ¿De acuerdo a los hallazgos encontrados cuales fueron las principales intervenciones de acompañamiento Psico-afectivo?

- Senderismo asistido, creación de grupos de amigos contemporáneos, actividad recreativa y de compartir.

- Competencia asistida y premiación como una actividad recreativa, cognitiva y Psico-afectiva.

\section{Discusión}

\section{Categoría 1: Practica Académica}

En cuanto a la práctica académica los académicos reconocemos que tradicionalmente se han explotado técnicas enfocadas al desarrollo teórico o de contenidos, siendo las más usadas expositivas, teórica - conceptual, conferencias, aprendizaje basado en problemas hipotéticos, memorística y repetitiva, en su mayoría expuestas desde los programas de asignaturas, en este sentido nos encontramos con un programa de la 
asignatura de "Cuidados Geriátricos" que orienta a un practica aplicada pero de forma pasiva, orienta una visita en el asilo de anciano con la finalidad de aplicar la valoración geriátrica para que los estudiantes logren el dominio teórico practico para aplicación de la historia clínica general del adulto mayor la que no va más allá de una técnica de aprendizaje basada en problemas, por tanto nuestra estrategia fue trasladada hasta la comunidad a través de un proyecto de seguimiento, esta estrategia fue desarrollado durante un semestre, contenía una planificación estratégica con una visión de tres vía:

1. Practica académica extensionista universidad - comunidad.

2. Vincular a los estudiantes en la solución de los problemas reales de la población.

3. Lograr un impacto positivo con las intervenciones de manera que resolvieran un problema real en la población seleccionada.

\section{Categoría 2: Valoración Físico - Psico-afectivo en el adulto mayor}

Habiendo pasado por un proceso de planificación siguiendo las pautas orientadas en el programa de la asignatura en mención, partimos del reconocimiento de esta población vulnerable, de acuerdo con Organización Mundial de la Salud (OMS) se considera adulto mayor a cualquier persona mayor de 60 años, de los cuales en su mayoría con problemas naturales que conlleva el envejecimiento, pero que necesitan ayuda para llevar a cabo sus actividades cotidianas por lo tanto requieren una constante observación y atención física, social y psicológica, esta organización manifiesta que se ha incrementado el número de padecimientos y sus complicaciones, con predominio de las enfermedades crónico degenerativas, deteriorando la calidad de la misma de los adultos mayores.

Los cambios en esta edad pueden ser negativo o positivo, según estilo de vida, por esto requiere de un cuidado multidisciplinario efectivo que incluya no sólo la recuperación sino también, muy destacadamente, la promoción de su salud. La enfermería se inserta de esta manera con su propia perspectiva en el cuidado integral del adulto mayor por lo que es necesario fortalecer los fundamentos que sustentan el cuidado humanizado del adulto mayor y la formación técnico-profesional de sus cuidadores.

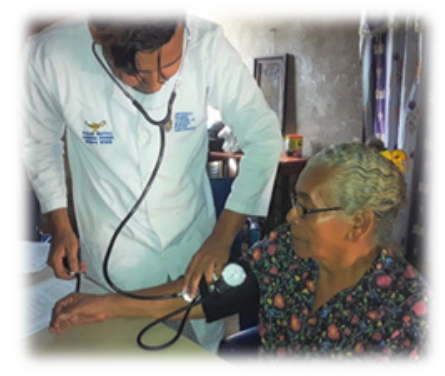


Ante esta premisa para ejecutar la estrategia de ayuda al adulto mayor, el método utilizado en las tres cohortes fueron en principio aplicar las guías de valoración geriátrica del adulto mayor, Guía del examen físico general, valoración de las actividades de la vidas diarias - índice de Kartz para el manejo de la dependencia y la independencia, Escala de Lawton Brody para las actividades instrumentales de la vida diaria, Valoración Mini Nutricional Assessment Nestlé, Escala Geriátrica de la depresión, todas estas establecidas como estándar en la literatura.

\section{Categoría 3: Evidencia In situ}

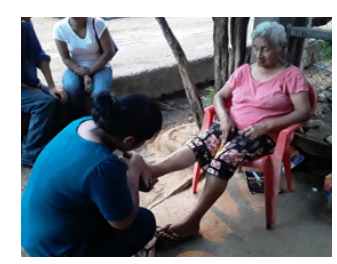

En cuanto a las manifestaciones de los problemas físicos encontrados en el adulto mayor, durante las tres cohortes los más sentidos están representados por la movilidad, deterioro de las estructuras óseas, problemas circulatorios, daños por problemas de enfermedades crónicas degenerativas como artritis, diabetes. Según Denham-Harman (1956) en su teoría de radicales libre menciona que el envejecimiento es resultado de los efectos perjudiciales fortuitos causados a tejidos por reacciones de radicales libres. Estas reacciones pueden estar implicadas en la producción de los cambios del envejecimiento, asociados con el medio ambiente, enfermedad y con su proceso intrínseco (Pardo, 2003).

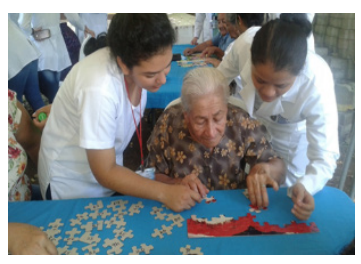

A nivel cognitivo la salud del adulto mayor se evidencio que había afectación marcada en las tres cohortes, cuyas manifestaciones estaban representadas en pérdida de la memoria a corto plazo, lagunas mentales y síndrome confuso a la hora de la valoración, las escalas no fueron completadas ya que olvidaron algunos elementos de su vida diaria. Según la teoría del enlentecimiento (Cerella, 1985; Salthouse, 1996), la declinación en el desempeño cognitivo se produce por un enlentecimiento general de la velocidad de la transmisión neural. Respaldan este enfoque Junqué y Jodar, (1990) con los hallazgos empíricos que muestran que las medidas de velocidad motora-perceptiva comparten mucha de la varianza asociada a la mayor edad en el desempeño de un amplio rango de tareas cognitivas (Véliz, \& Arancibia, 2010). 
A nivel Psico - Afectivo la salud del adulto mayor se vio afectada en las tres cohortes, siendo los más sentidos la soledad, tristeza, abandono y dependencia progresiva, a pesar de la responsabilidad por parte de los tutores. Barros (2008) Manifiesta que la teoría de la desvinculación establece que la separación de las personas y sus vínculos emocionales se da a partir de la concepción de ellas mismas como individuos incapaces, esto quiere decir que es la sociedad quien los excluye por motivos como la dependencia de las actividades básicas y enfermedad (Ordoñez, \& Ruiz, 2015).

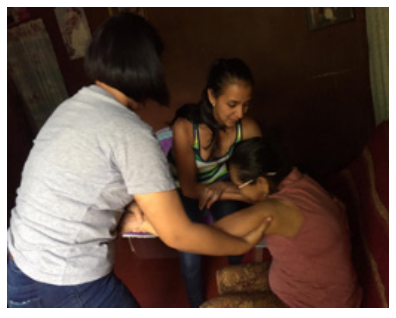

De acuerdo a los resultados encontrados en la valoración logramos establecer intervenciones, desde el punto de vista motora realizamos la aplicación y enseñanza de ejercicios físicos con el objetivo de ayudar a la estimulación de la deambulación y el reposo, siendo esta de gran beneficio para los adultos mayores.

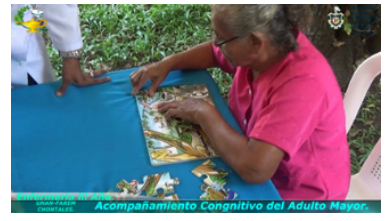

A nivel Cognitivo logramos intervenir aplicando estrategias de estimulación del recuerdo, con el objetivo de mejorar su agilidad cognitiva, aclarar lagunas mentales y síndrome confuso.

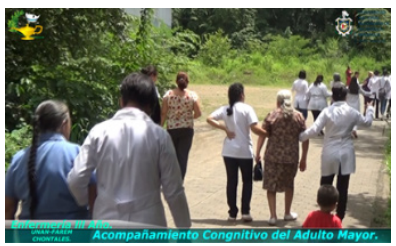

En cuanto a las intervenciones de acompañamiento Psico - afectivo realizamos senderismo asistido, actividades recreativas y formación de club de amigos contemporáneos, para mejorar su estado emocional y así aportar a la inclusión social de igual manera realizamos competencia asistida y premiación como actividad recreativa cognitiva y Psico- afectiva. 


\section{Conclusiones y perspectivas futuras}

Es menester detallar aspectos esenciales en la aplicación de una estrategia de ayuda desde la extensión universitaria, logrando una participación activa de los estudiantes de la carrera de enfermería orientado al desarrollo de habilidades técnicas, científicas y humanísticas, pero además una integración de la universidad con la comunidad, acompañando acciones que garantizan el cambio en la calidad de vida de una población vulnerables.

En la primera cohorte 2018 logramos establecer la estrategia dirigida a población vulnerable con vinculo cercano con los estudiantes, se apertura la estrategia con 18 adultos mayores de los cuales solo lograron culminar el periodo de asistencia 15 el resto algunos familiares no dieron apertura a las visitas en el hogar, en este periodo se logró documentar los tres elementos estratégicos planteados anteriormente. La evaluación de la estrategia fue cumplida en un $85 \%$ por interrupciones en el tiempo, sin embargo los estudiantes lograron la competencia de valoración, diagnostico, planificación y ejecución de las intervenciones. Los adultos mayores asistidos manifestaron mejoría en su salud física y emocional pero también los familiares valoraron como positivo el acompañamiento dirigido, otros sectores de la población solicitaron la continuidad de la estrategia por su capacidad resolutiva desde la universidad.

En el segundo cohorte 2019 se repite la estrategia con nuevos protagonistas que tenían las mismas características, al mismo tiempo se agregaron otras intervenciones en el plan de ayuda que no fueron incluidas en la primera cohorte porque no se completaron todas las escalas de valoración, en este año se asistieron un total de 15 adultos mayores ninguno de ellos deserto de la estrategia, se dio adiestramiento a los familiares para la continuidad de la estrategia. En este mismo año se logro un cumplimiento del 100\% en la aplicación de la estrategia desde su planificación didáctica hasta la documentación de los resultados, logramos observar que en las dos cohortes ya teníamos un número significativo de adultos mayores en asistencia domiciliar porque los primeros 15 ya eran asistidos por sus familiares quienes recibieron adiestramiento por los estudiantes, los que se han estado monitoreados a través de una base de datos.

En la tercer cohorte 2020 se aplica nuevamente la estrategia, se continua la misma metodología pero igual se ingresan nuevos protagonistas al programa de asistencia dirigida con 15 nuevos adultos mayores y sus representantes tutores que se sumaron al adiestramiento para el auto cuidado, la estrategia fue un éxito la comunidad ha reconocido el impacto positivo de esta acción social extensionista de la universidad, cada vez se suman nuevos protagonistas a esta acción que se convierte en una esperanza para el mejoramiento de la calidad de vida del adulto mayor. Aunque el contexto de la pandemia de la Covid-19 ha puesto en mayor estado de vulnerabilidad la salud del adulto mayor es meritorio reconocer que los 45 adultos mayores asistidos 
en el hogar en las tres cohortes se mantienen estable a pesar de problemas coránicos de la edad, mismos que están siendo asistidos por sus familiares.

Para nosotros es satisfactorio declarar este beneficio como una respuesta de impacto positivo ante una estrategia de ayuda propuesta, dirigida y ejecutada desde una actividad académica- formativa de la Universidad Nacional Autónoma de Nicaragua FAREM- Chontales, Departamento de Ciencia tecnología y salud. Misma que debería continuar aplicándose haciendo ajustes en el tiempo y contexto.

\section{Lista de referencia}

Cedeño. F, J. \& Machado. R, E.F. (2012). Papel de la Extensión Universitaria en la transformación local y el desarrollo social. En https://bit.ly/3wbCUKs

Di Mari, D. (2013). La Importancia de la Extensión Universitaria: Anuario Escuela de Archivología IV - Universidad de Córdoba. En https://bit.ly/2SIzoVf

Indra. (2012). Forlab - Forensic Laboratory for in-situ evidence analysis in a post blast scenario: Proyectos de innovación.

Menéndez, G. (2017). Desarrollo y conceptualización de la Extensión Universitaria. Un aporte de la secretaría de Extensión de la Universidad Nacional del Litoral para el análisis y debate de la Extensión Universitaria. En https://bit.ly/33NtAQF

Ministerio de educación Guatemala. (2010). Metodología del Aprendizaje: Currículo organizado en Competencias. Primera Edición. En https://bit.ly/3hwP $4 c \mathrm{C}$

Melendro, M. (2011). Metodología de la intervención: procesos y estrategias, en Pérez Serrano (Coord.) Intervención Socioecomunitaria (pp. 569-588). Madrid: UNED En https://bit.ly/3wbwk6s

Organización de las Naciones Unidas para la Agricultura y la Alimentación (FAO). (2004). Guía Metodológica para la Sistematización: Programa especial para la seguridad alimentaria PESA en Centro America. En http://www.fao.org/3/at773s/ at773s.pdf

Organización Mundial de la Salud (2018): Envejecimiento y Salud. Tomado de: https:// www.who.int/es/news-room/fact-sheets/detail/envejecimiento-y-salud. El 19 de mayo 2021.

Pérez de Maza, T. (2016). Sistematización de experiencias en contextos universitarios: Guía Didáctica. Caracas: Universidad Nacional Abierta, Ediciones del Vicerrectorado Académico, primera edición. En https://bit.ly/3w4d4bo 
Pilar, G. M. (2015). Escuela Nueva Vs Escuela Tradicional. Revisado en: red educa.net En https://bit.ly/3bm2NiH

Pardo Andreu, Gilberto. (2003). Consideraciones generales sobre algunas de las teorías del envejecimiento. Revista Cubana de Investigaciones Biomédicas, 22(1) Recuperado en 19 de mayo de 2021, de http://scielo.sld.cu/scielo. php?script=sci_arttext\&pid=So864-03002003000100008\&lng=es\&tlng=es

Pérez, T.V., Blanco, E. E. E, \& Gómez, G. G. (2017). Estrategias pedagógicas en el aula de clases. Revista Colombiana de Tecnologías de Avanzada (RCTA), 2(28), 124-131.

Quintero-Rodríguez, M., Victorio-Quintero, M., \& Botero-Caicedo, Y.A. (2015). Tranformaciones de subjetividad generadas en la integración psicosocial entre el "padrino afectivo" y el adulto mayor institucionalizado. Criterio Libre Juridico, 12(2), $151-159$.

Vásquez. R, F. (2010). Estrategias de enseñanza: Investigaciones sobre didáctica en instituciones educativas de la ciudad de Pasto, Bogotá D.C. Editorial: Kimpres, Universidad de la Salle En https://bit.ly/3fkkbp7

Valenzuela Tovar, S.M. (2018). Union Latinoamericana de Extención Universitaria: un espacio de cooperación, intercambio y refelxión en materia de extención y vinculación con la sociedad. +E: Revista de Extención Universitaria, 8(8), enero - junio, 61-88 doi: 10.14409/ extensión.v88.Ene-Jun. 7720.

Véliz, Mónica, Riffo, Bernardo, \& Arancibia, Beatriz. (2010). Envejecimiento Cognitivo Y Procesamiento Del Lenguaje: Cuestiones Relevantes. Rla. Revista de lingüística teórica y aplicada, 48(1), 75-103. https://dx.doi.org/10.4067/ So718-48832010000100005

Zavala, A. U. R. O. R. A. (2010). La sistematización desde una mirada interpretativa: Propuesta metodológica. Trabajo social UNAM. VI Época, 1. En http://revistas. unam.mx/index.php/ents/article/view/23884 